\title{
Pré-natal odontológico: bases científicas para o tratamento odontológico durante a gravidez
}

Dental prenatal care: scientific basis for dental treatment during pregnancy

Atención odontológica prenatal: base científica para el tratamiento odontológico durante el embarazo Priscilla Ramos PEREIRA ${ }^{1}$

Agnes ASSAO ${ }^{1}$

Andréa Lemos Falcão PROCóPIO'

Juliana Moura Storniolo de SOUZA ${ }^{1}$ Marina Ciccone GIACOMINI

Priscilla Santana Pinto GONÇALVES ${ }^{1}$

Gerson Aparecido FORATORI-JUNIOR ${ }^{2}$

${ }^{1}$ Faculdade de Odontologia, UNIFIO Centro Universitários da Faculdades Integradas de Ourinhos, 19909-100 Ourinhos - SP, Brasil

${ }^{2}$ Departamento de Odontopediatria, Ortodontia e Saúde Coletiva, Faculdade de Odontologia de Bauru, USP Universidade de São Paulo, 17012-901 Bauru - SP, Brasil

\section{Resumo}

Ainda existem muitos tabus na sociedade sobre o acompanhamento odontológico durante a gestação e que, somado ao desconhecimento por parte dos profissionais, dificultam a difusão do conceito de pré-natal odontológico. O objetivo deste estudo foi sintetizar as principais evidências acerca do manejo da mulher durante a gestação, apontando as principais condutas que os profissionais devem adotar no estabelecimento de um protocolo holístico de pré-natal odontológico. No período de outubro/2020 a março/2021, as seguintes bases de dados foram acessadas: EMBASE, SCOPUS, MEDLINE (Entrez PubMed), Web of Science, BVS/LILACS, Cochrane Library e SCIELO. Foram incluídos estudos publicados entre janeiro/2000 a março/2021 em espanhol, inglês e português. O atendimento odontológico durante a gravidez é seguro e recomendado e os protocolos odontológicos preventivos devem ser adotados à todas as gestantes. Os procedimentos odontológicos clínicos podem ser realizados durante toda a gestação quando necessários, desde que existam justificativas plausíveis. Os profissionais devem realizar a anamnese e o exame clínico de maneira minuciosa a fim de evitar a exposição desnecessária das pacientes aos exames radiográficos e ao sobretratamento. É de suma importância que os cirurgiões-dentistas conheçam as indicações e contraindicações da radiografia odontológica, terapia medicamentosa e uso de anestésicos locais na gravidez, a fim de atuar com segurança, garantindo saúde para as mulheres e seus filhos.

Descritores: Cuidado Pré-Natal; Gravidez; Odontologia Preventiva; Saúde Bucal.

\section{Abstract}

There are still many taboos in society about dental care during pregnancy and which, in addition to the lack of knowledge by professionals, make it difficult to spread the concept of dental prenatal care. The aim of this study was to synthesize the main evidence about the management of pregnant women, pointing out the main conducts that professionals should adopt in the establishment of a holistic dental prenatal protocol. From October/2020 to March/2021, the following databases were accessed: EMBASE, SCOPUS, MEDLINE (Entrez PubMed), Web of Science, BVS/LILACS, Cochrane Library and SCIELO. Studies published between January/2000 to March/2021 in Spanish, English and Portuguese were included. Dental care during pregnancy is safe and recommended and preventive dental protocols must be adopted for all pregnant women. Clinical dental procedures can be performed throughout pregnancy when necessary, as long as there are plausible justifications. Professionals should conduct anamnesis and clinical examination in order to avoid unnecessary exposure of patients to radiographic examinations and overtreatment. It is extremely important that dentists know the indications and contraindications of dental radiography, drug therapy and the use of local anesthetics during pregnancy, in order to act safely, ensuring health for women and their children.

Descriptors: Prenatal Care; Pregnancy; Preventive Dentistry; Oral Health.

\section{Resumen}

Aún existen muchos tabúes en la sociedad sobre el cuidado dental durante el embarazo y que, además del desconocimiento por parte de los profesionales, dificultan la difusión del concepto de atención odontológica prenatal. El objetivo de este estudio fue sintetizar las principales evidencias sobre el manejo de la gestante, señalando las principales conductas que deben adoptar los profesionales en el establecimiento de un protocolo prenatal dental holístico. Desde octubre/2020 hasta marzo/2021, se accedió a las siguientes bases de datos: EMBASE, SCOPUS, MEDLINE (Entrez PubMed), Web of Science, BVS/LILACS, Cochrane Library y SCIELO. Se incluyeron estudios publicados entre enero/2000 a marzo/2021 en español, inglés y portugués. El cuidado dental durante el embarazo es seguro y recomendado y se deben adoptar protocolos dentales preventivos para todas las embarazadas. Los procedimientos clínicos dentales se pueden realizar durante todo el embarazo cuando sea necesario, siempre que existan justificaciones plausibles. Los profesionales deben realizar la anamnesis y el examen clínico de manera adecuada para evitar la exposición innecesaria de los pacientes a exámenes radiográficos y sobretratamiento. Es sumamente importante que los odontólogos conozcan las indicaciones y contraindicaciones de la radiografía dental, la farmacoterapia y el uso de anestésicos locales durante el embarazo, para poder actuar de forma segura, garantizando la salud de la mujer y sus hijos.

Descriptores: Atención Prenatal; Embarazo; Odontología Preventiva; Salud Bucal.

INTRODUÇÃO

O pré-natal é considerado um momento ideal para que intervenções de saúde sejam realizadas, visto que nesse momento a mulher encontra-se aberta a adquirir novos conhecimentos, com o objetivo de proporcionar melhor cuidado de saúde para o bebê. Devemos considerar ainda que ao receber as orientações de saúde durante a gestação, a mulher poderá agir como instrumento de propagação da informação para os demais membros da família. Desta forma, é de extrema importância que estratégias bem delineadas de educação em saúde ocorram no período gestacional ${ }^{1,2}$.

Frente às alterações fisiológicas, principalmente as imunológicas e hormonais, estudos têm associado a gravidez com as doenças periodontais ${ }^{3}$. A alta prevalência de gengivite na gestação relaciona-se à resposta aguda ao biofilme, uma vez que essas mulheres 
estão mais propensas à inflamação decorrente dos altos níveis de estrógeno e progesterona e, consequentemente, redução da resposta imune ${ }^{4}$.

O pré-natal odontológico faz menção ao acompanhamento que visa orientar as gestantes sobre os cuidados com a própria saúde bucal e sanar as demandas bucais dessas gestantes, uma vez que tais problemas colocam as mulheres e seus bebês ao maior risco de doenças ${ }^{3,5-7}$. A partir das estratégias de educação em saúde adotadas para as gestantes, espera-se reduzir o tabu que ainda persiste na sociedade acerca das indicações e contraindicações de determinados procedimentos e condutas no âmbito odontológico.

As principais barreiras que impedem a difusão da prática do pré-natal odontológico consistem em: 1) historicamente, as mulheres durante o período gestacional ficaram à margem dos serviços odontológicos no setor público de saúde, tendo acesso apenas aos atendimentos de urgência ${ }^{8}$. Panorama este que tem mudado desde a criação do Programa Brasil Sorridente ${ }^{9}$. 2) a integração entre a Odontologia e a comunidade por anos foi precária, baseada muitas vezes em seu caráter assistencialista, o que resultou em maior confiabilidade das mulheres para com os médicos, os quais, por sua vez, desconheciam a necessidade do acompanhamento odontológico durante a gestação ou conhecem as necessidades, mas não adotam as medidas cabíveis ${ }^{10,11}$; 3 ) a falta de conhecimento sobre a temática dos cirurgiões-dentistas reforçava os tabus da própria sociedade, gerando insegurança dos próprios profissionais no manejo das mulheres durante a gravidez. Acerca desta, ressalta-se a necessidade de reestruturação das matrizes curriculares das instituições de ensino Superior a fim de contribuir com a melhor formação e capacitação dos alunos para serem aptos ao atendimento das gestantes ${ }^{8}$.

Diante do exposto, o objetivo deste estudo foi sintetizar as principais evidências acerca do manejo da mulher durante a gestação, apontando as principais condutas que os profissionais devem adotar no estabelecimento de um protocolo holístico de pré-natal odontológico.

MATERIAL E MÉTODO

- Paciente e Envolvimento Público

O presente estudo trata-se de uma análise de dados secundários, por meio de uma revisão de literatura do tipo narrativa, uma vez que compõe uma análise da literatura publicada sobre o cuidado holístico durante o pré-natal odontológico. Desta forma, esta revisão de literatura foi realizada sem envolvimento do paciente. Os pacientes não foram envolvidos de forma alguma durante a revisão da literatura disponível, nem durante a redação ou edição deste documento.

- Estratégia de busca

No período de outubro/2020 a março/2021, as seguintes bases de dados foram acessadas: EMBASE (https://www.embase.com), $\quad$ SCOPUS (https://www.scopus.com), MEDLINE (Entrez PubMed, https://www.ncbi.nim.nih.gov), Web of Science (https://www.webofscience.com), BVS/LILACS (https://lilacs.bvsalud.org), Cochrane Library (https://www.cochranelibrary.com) e SCIELO (https://scielo.org), considerando as seguintes palavras-chave: (pregnancy OR pregnant woman OR prenatal care) AND (dentistry OR general practice, dental OR preventive dentistry OR practice patterns, dentists' OR partnership practice, dental) AND (oral health OR dental caries OR dental erosion OR gingivitis OR periodontitis OR chronic periodontitis OR aggressive periodontitis), as quais foram ajustadas para cada uma das bases de dados. Os respectivos termos das palavras-chave citadas anteriormente em português e espanhol também foram acessados.

- Critérios de elegibilidade

Foram selecionados nesta revisão de literatura estudos publicados entre janeiro/2000 e março/2021, nos idiomas português, inglês e espanhol. Dentre eles, foram considerados: clínicos intervencionais (ensaios clínicos randomizados e não randomizados; e não-cego, duplo-cego ou triplo-cego) e observacionais (transversais, coortes e de caso-controle). Outras revisões de literatura (sistemática com e sem meta-análise; ou não sistemática) foram incluídas. Não foram considerados nesta revisão de literatura os relatos de caso, séries de casos, artigos de opinião, editoriais, cartas aos editores, estudos laboratoriais envolvendo animais, protocolos metodológicos e resumos de eventos científicos (expandidos ou não expandidos).

RESULTADOS E DISCUSSÃO

O pré-natal odontológico é importante para medir e prever possíveis problemas, prescrever medicamentos e realizar cuidados dentários durante os exames radiográficos, induzindo um tratamento seguro e eficaz e reduzindo o risco de efeitos prejudiciais para o bebê $^{12,13}$. É de extrema importância que os cirurgiões-dentistas se sintam seguros para o atedimento odontológico de gestantes. 
Apesar do primeiro trimestre de gestação ser o mais crítico, visto que o feto é mais suscetível a efeitos teratogênicos e aborto espontâneo, os profissionais devem compreender que as mulheres podem ser atendidas durante toda a gestação. $O$ segundo trimestre é o mais indicado para tratamentos odontológicos eletivos, já que evita a fase de organogênese ( $1^{\circ}$ trimestre) e também evita 0 período final da gestação ( $3^{\circ}$ trimestre) em que há maior desconforto da mãe para o adequado posicionamento na cadeira odontológica ${ }^{12}$.

No terceiro trimestre, devido a posição anatômica do feto no corpo da mulher, o risco de síncope e hipertensão é maior, assim como as necessidades cardiovasculares e respiratórias também são maiores. Recomendase, portanto, consultas curtas, evitando 0 período da manhã devido aos enjoos recorrentes e o profissional deve sempre manter a paciente adequadamente posicionada na cadeira odontológica (posição semideitada). Deve-se evitar a posição supina da cadeira odontológica, uma vez que nessas situações é comum que ocorra a compressão da veia cava inferior, prejudicando o retorno venoso e a oxigenação das mulheres, as quais podem apresentar tonturas e náuseas (síncope da veia cava inferior). Como o retorno venoso da veia cava inferior ocorre pelo lado direito, recomenda-se sempre orientar as pacientes a permanecerem ligeiramente inclinadas para 0 lado esquerdo, facilitando o retorno venoso e prevenindo a ocorrência da síncope ${ }^{12,14}$.

Existe um tabu na Odontologia, tanto para a população leiga quanto para alguns profissionais, acerca da realização de radiografias dentárias em mulheres grávidas. Em geral, acredita-se que a radiografia deve ser evitada no primeiro trimestre de gestação, principalmente entre a $4^{\underline{a}}$ e $5^{\text {a }}$ semanas de gestação, devido a organogênese ser um momento crítico. Porém, se todas as medidas preventivas forem adequadamente adotadas, como uso de avental plumbífero com chumbo e ajuste de dose e duração dos raios-X, não há necessidade de evitar ou adiar os exames radiológicos para o pós-parto, principalmente em situações de urgência odontológica ${ }^{12,15,16}$. Os profissionais devem realizar uma anamnese e exame clínico detalhado para evitar ao máximo o erro de técnica, a fim de reduzir as repetições e exposições desnecessárias aos raios-X. Ademais, os profissionais podem lançar mão de filmes/sensores ultrassensíveis, reduzindo o tempo de exposição das pacientes aos raios- $X^{12,15,16}$.

Considera-se que a dose recebida pelo feto nas radiografias odontológicas é muito menor do que a dose que poderia causar malformações congênitas (aproximadamente 10 $\mathrm{mSv}$ ), visto que o feto absorve apenas $1 / 50000$ da exposição direta dos raios- $X$ na cabeça da mãe. Portanto, se houver a necessidade de realizar a radiografia odontológica como exame complementar durante a gestação, os profissionais podem realizar desde que haja justificativas plausíveis ${ }^{12,15,16}$. No quadro 1 é possível observar as doses de radiação resultantes de diferentes tomadas radiográficas comumente indicadas na Odontologia.

Quadro 1. Doses de radiação das tomadas radiográficas odontológicas

\begin{tabular}{|l|c|}
\hline \multicolumn{1}{|c|}{ RADIOGRAFIAS } & DOSES DE RADIAÇÃO \\
\hline Periapical $\backslash$ Interproximal (bitewing) & 0,001 a $0,008 \mathrm{mSv}$ \\
\hline Panorâmica & 0,026 a $0,030 \mathrm{mSv}$ \\
\hline Tomografia Computadorizada Cone Beam da Face & $\approx 0,034$ a $0,652 \mathrm{mSv}$ \\
\hline
\end{tabular}

Durante a gestação é comum as mulheres apresentarem episódios de vômitos, principalmente no início da gravidez. Além dos episódios erosivos, é comum que as pacientes realizam escovação dos dentes após os vômitos, associando 0 desgaste dentário erosivo à abrasão dentária, o que pode resultar na perda de estrutura mineral dos elementos dentários. No entanto, é importante ressaltar que não há evidências plausíveis que associem o desgaste dentário erosivo com a gestação, uma vez que 0 desgaste dentário erosivo/biocorrosão é caracterizado por um processo tempo-dependente. Desta forma, episódios isolados de vômitos, associados ou não à abrasão dentária, não são capazes de ocasionar perda de estrutura mineral dos dentes quando o indivíduo apresentar equilíbrio dos componentes salivares e do processo de desmineralização-remineralização ${ }^{17}$. Entretanto, diante do risco de ocorrência, é de extrema importância que os profissionais tenham o conhecimento adequado e os pacientes sejam orientados da conduta a ser adotada frente aos episódios erosivos durante a gravidez.

Algumas mulheres grávidas podem apresentar a sensação de boca seca (xerostomia) de maneira temporária, o que pode ser explicado por alterações hormonais relacionadas à gravidez. Consumir água e balas ou goma de mascar sem açúcar com mais frequência pode aliviar esse problema. Em casos mais severos, recomenda-se o uso de saliva artificial. Também é recomendado que mulheres com xerostomia sejam expostas com mais frequência ao flúor (dentifrício, enxaguatório bucal), evitando o processo de desmineralização e reduzindo o risco de cárie dentária ${ }^{15}$.

O uso de flúor durante a gravidez só é 
recomendado topicamente para melhorar a saúde bucal de gestantes com médio e alto risco à cárie dentária. Não se recomenda a administração de suplementos vitamínicos contendo flúor, uma vez que, além de não contribuir na prevenção da cárie dentária para as mulheres e para as crianças, a presença do flúor no composto reduz consideravelmente a absorção de cálcio do complexo vitamínico, prejudicando a saúde das mulheres, visto que o cálcio é um composto fundamental a ser reposto na gravidez ${ }^{18}$.

Frente as alterações metabólicas e fisiológicas que ocorrem durante a gestação, as doenças periodontais são muito prevalentes durante a gravide ${ }^{19}$, podendo estar associada a outros problemas de saúde sistêmico das mulheres. Esses problemas bucais podem produzir dor e complicações e podem exigir intervenções mais complexas caso não sejam tratadas rapidamente. Outra consequência bucal durante a gestação é o tumor gravídico, o qual acomete até $5 \%$ das gestantes. Essa lesão vascular hiperplásica é causada a partir do aumento hormonal e presença de paca bacteriana no local. As lesões são tipicamente eritematosas, moles e lobuladas, encontrandose principalmente na gengiva. A mucosa da língua, o palato ou a mucosa bucal também podem estar envolvidos. Os tumores gravídicos são mais comuns após o primeiro trimestre, evoluem rapidamente e normalmente regridem após o parto. O protocolo de tratamento recomendado é o acompanhamento, a menos que os tumores apresentem sangramento, interfiram na mastigação ou não tenham regressão espontânea após o parto. Lesões removidas cirurgicamente durante a gravidez têm maior risco de recidiva dentro do período gestacional $^{4,20}$.

Diante da elevada prevalência de alterações nos tecidos periodontais durante a gravidez, se faz necessário que os cirurgiõesdentistas adotem protocolos bem estabelecidos de prevenção em odontologia. No pré-natal odontológico é recomendado que as pacientes façam profilaxias nos dentes e as orientações de higiene sejam reforçadas mensalmente, quanto à quantidade e qualidade das escovações dentária, bem como sobre o uso do fio dental diariamente. Quando necessário, as pacientes devem passar por terapia periodontal de suporte (raspagem supra e/ou subgengival) mensalmente ou bimensalmente. Estudos prévios mostram que a terapia periodontal de suporte durante a gestação melhora os parâmetros de saúde das mulheres e reduz o risco de mortalidade e prematuridade do bebê ${ }^{21}$.
Diversas são as dúvidas dos profissionais da odontologia a respeito da terapia medicamentosa durante a gestação. Nas décadas de 1950 e 1960, devido ao aumento da incidência de bebês com deformidades nos membros e ao uso da talidomida (um medicamento anteriormente usado para aliviar os enjoos matinais), as pesquisas sobre medicamentos durante a gravidez tornaram-se significativas. Considerando a capacidade de difusão de várias drogas através da membrana placentária, a principal preocupação das gestantes durante o tratamento medicamentoso é evitar os efeitos teratogênicos ${ }^{12,15}$.

Para determinar os riscos associados ao uso de medicamentos durante a gravidez e orientar os dentistas sobre o tratamento mais adequado para a gestante, a organização do FDA (Food and Drug Administration) estabeleceu uma classificação que é utilizada desde 1979, onde categoriza os medicamentos em cinco grupos de risco, levando em consideração seu impacto na gravidez, sendo: A) estudos controlados em humanos não indicam riscos aparentes para o feto; B) estudos em animais não indicam risco para o feto, mas ainda sem estudos confiáveis com mulheres grávidas; C) estudos em animais mostraram efeitos adversos para o feto, mas não existem estudos em humanos; D) evidências positivas de risco fetal humano, mas cujos benefícios podem justificar o uso; X) Evidências positivas de anormalidades fetais, com contraindicações tanto em mulheres grávidas quanto nas que querem engravidar, pois os riscos superam os benefícios. De acordo com esta classificação, os medicamentos das classes A e B podem ser prescritos com segurança para mulheres grávidas, e os medicamentos das classes C e D devem ser prescritos apenas quando estritamente necessário. Por outro lado, os medicamentos classificados como $X$ não devem ser usados em nenhuma circunstância. Levando em consideração as recomendações da OMS, é fundamental que o dentista informe e explique ao paciente a finalidade do tratamento, a duração da medicação, seus benefícios e riscos, e as condutas a serem seguidas em caso de condições adversas ${ }^{12,15,22}$. Ademais, é sempre indicado que os cirurgiões-dentistas contatem os médicos das pacientes que são responsáveis pelo pré-natal, informando-os da necessidade de administração das determinadas drogas.

Nos quadros 2 e 3 estão apresentados os principais medicamentos (analgésicos/antiinflamatórios e antibióticos, respectivamente) que são indicados e contraindicados durante a 
gestação, de acordo com a classificação da FDA.

Quadro 2. Recomendação medicamentosa durante a gravidez (analgésicos/anti-inflamatórios)

\begin{tabular}{|c|c|}
\hline Analgésicos & Recomendação \\
\hline $\begin{array}{c}\text { Acetaminofeno, Acetaminofeno } \\
\text { com codeína; hidrocodona ou } \\
\text { oxicodona, Codeína, Meperidina, } \\
\text { Morfina }\end{array}$ & Podem ser usados durante a gestação \\
\hline Aspirina, Ibuprofeno, Naproxeno & $\begin{array}{c}\text { Podem ser usados em curta duração } \\
\text { durante a gestação }(48-72 \text { horas). } \\
\text { Porém, evitar nos } 1^{\circ} \text { e } 3^{\circ} \text { trimestres }\end{array}$ \\
\hline
\end{tabular}

Quadro 3. Recomendação medicamentosa durante a gravidez (antibióticos)

\begin{tabular}{|c|c|}
\hline Antibióticos & Recomendação \\
\hline $\begin{array}{c}\text { Amoxicilina, Cefalosporina, } \\
\text { Clindamicina, Metronidazol* } \\
\text { Penicilina }\end{array}$ & $\begin{array}{c}\text { Podem ser usados durante a gestação } \\
\text { * Evitar o Metronidazol no } 1^{\circ} \\
\text { trimestre }\end{array}$ \\
\hline $\begin{array}{c}\text { Ciprofloxacina, Claritromicina, } \\
\text { Levofloxacina, Moxifloxacina }\end{array}$ & Evitar o uso durante a gestação \\
\hline Tetraciclina, Eritromicina Estolato & $\begin{array}{l}\text { Nunca devem ser usados durante a } \\
\text { gestação }\end{array}$ \\
\hline
\end{tabular}

A indicação dos anti-inflamatórios não esteroidais (AINEs) e do ácido acetilsalicílico deve ser cautelosa na gestação, pois podem causar sangramento na mãe e no feto. Além disso, devido à inibição da síntese de prostaglandinas relacionada às contrações uterinas, o uso de AINEs no último trimestre da gravidez geralmente leva a partos prolongados $^{12,15,22}$.

Evidências prévias associam a administração de anti-inflamatórios não esteroidais à teratogenicidade. ${ }^{12,15,22}$ Por exemplo, a aspirina parece estar associada à hipertensão pulmonar fetal e retardo do crescimento fetal, enquanto o ibuprofeno pode estar associado a efeitos nocivos no feto e na circulação neonatal $^{12,15,22}$.

O paracetamol é um analgésico (Classe B) que, quando usado em doses terapêuticas, não tem efeitos teratogênicos e pode ser usado com segurança para tratar dores leves a moderadas em qualquer estágio da gravidez, sendo, portanto, os analgésicos mais adequados para mulheres grávidas que estão disponíveis no mercado ${ }^{12,15,22}$.

Em algumas situações, os corticosteroides classificados como Categoria C de acordo com a classificação da FDA são considerados mais seguros do que os AINEs, quando administrados localmente. Nos casos em que esses medicamentos são necessários, prednisona ou prednisolona devem ser os medicamentos de primeira escolha, devido ao menor risco de efeitos adversos no feto devido à dificuldade de passagem pela membrana placentária ${ }^{12,15,22}$. Segundo Poletto et al. ${ }^{23}$, em caso de cirurgia ou intervenção endodôntica que não possa ser postergada, o melhor é o uso de corticosteroides em vez de AINEs.

Para prevenir e tratar infecções bucais durante a gravidez, o antibiótico de escolha é a penicilina. Por ter efeito específico sobre as substâncias da parede celular bacteriana, não causa prejuízos à mãe ou ao feto. Amoxicilina e Ampicilina (Classe B) são os mais indicados, cefalosporinas e macrolídeos são outras opções pertencentes ao mesmo grupo (categoria B). Devido à sua hepatotoxicidade, a Eritromicina Estolato não deve ser ingerida ${ }^{12,15,22}$.

A Tetraciclina (Classe D) não é indicada porque penetra facilmente na membrana placentária e quela o cálcio no tecido dentário fetal durante 0 processo de mineralização, levando à malformação do esmalte e descoloração do dente. Além disso, esse medicamento também pode causar atrasos no desenvolvimento fetal. Em infecções mais graves, os pacientes alérgicos à penicilina podem escolher usar clindamicina $(600 \mathrm{mg})$ ou combinar a penicilina com metronidazol ${ }^{12,15,22}$. No entanto, alguns autores contraindicam o uso do Metronidazol no $1^{\circ}$ trimestre de gestação, devido aos possíveis efeitos adversos na formação do feto ${ }^{15}$.

Assim como a terapia medicamentosa, a administração de anestésicos locais em tratamentos odontológicos causa insegurança nos profissionais. Os anestésicos locais são totalmente seguros durante a gravidez e não há contraindicações ao uso ${ }^{15,24,25}$. A maioria dos anestésicos são considerados como Classe B, de acordo com a classificação da FDA, com exceção da Mepivacaína e da Bupivacaína (Classe C). Embora tenham a capacidade de cruzar a barreira placentária, não há evidências que os associem com efeitos teratogênicos. Os anestésicos mais comumente usados são lidocaína com $2 \%$ de epinefrina na concentração de 1:100.000 24 .

Não há contraindicações do uso de vasoconstritores na solução anestésica e suas vantagens se referem ao aumento da concentração local do anestésico (redução da toxicidade sistêmica), hemostasia e o prolongamento dos efeitos farmacológicos, proporcionando tempo adequado para as intervenções ${ }^{15,24,25}$. Ao realizar a anestesia local, a solução deve ser injetada lentamente e aspirada previamente como medida preventiva para evitar a injeção intravascular. Além disso, no máximo dois anestésicos $(3,6 \mathrm{ml})$ devem ser usados em cada sessão de tratamento, e a técnica anestésica deve ser usada corretamente $^{24}$.

É importante ressaltar que não se recomenda o uso de benzocaína e prilocaína como anestésicos locais, pois eles reduzem a circulação placentária e apresentam risco de metemoglobinemia e hipóxia fetal. E a solução 
contendo felipressina na composição deve ser evitada, uma vez que pode estimular a contração uterina devido a sua semelhança estrutural com a ocitocina em grandes doses $^{15,24,25}$.

CONCLUSÃO

Considerando tais evidências disponíveis acerca dos cuidados odontológicos durante 0 pré-natal, o atendimento odontológico durante a gravidez é seguro e recomendado. Protocolos odontológicos preventivos devem ser adotados às gestantes. Os procedimentos odontológicos clínicos podem ser realizados durante toda a gestação quando necessários, desde que existam justificativas plausíveis. Assim, a anamnese e o exame clínico devem ser bem conduzidos para evitar a exposição desnecessária das pacientes aos exames radiográficos e ao sobretratamento. É de suma importância que os cirurgiões-dentistas conheçam as indicações e contraindicações da radiografia odontológica, terapia medicamentosa e uso de anestésicos locais na gravidez, a fim de atuar com segurança, garantindo saúde para as mulheres e seus filhos. Por fim, é importante destacar que as matrizes curriculares dos cursos de Odontologia devem dar um maior enfoque ao pré-natal odontológico, a fim de que os graduandos já se sintam confiantes e aptos na inserção do mercado de trabalho e obtenham sucesso no tratamento odontológico de mulheres durante a gestação.

REFERÊNCIAS

1. Herval ÁM, Oliveira DPD, Gomes VE, Vargas AMD. Health education strategies targeting maternal and child health: A scoping review of educational methodologies. Medicine (Baltimore). 2019;98:e16174.

2. Liu P, Wen W, Yu KF, Gao X, Lo ECM, Wong MCM. Effectiveness of a family-centered behavioral and educational counselling approach to improve periodontal health of pregnant women: a randomized controlled trial. BMC Oral Health. 2020;20:284.

3. Komine-Aizawa S, Aizawa S, Hayakawa S. Periodontal diseases and adverse pregnancy outcomes. J Obstet Gynaecol Res. 2019;45:5-12.

4. Silva de Araujo Figueiredo C, Gonçalves Carvalho Rosalem C, Costa Cantanhede AL, Abreu Fonseca Thomaz ÉB, Fontoura Nogueira da Cruz MC. Systemic alterations and their oral manifestations in pregnant women. J Obstet Gynaecol Res. 2017;43:16-22.

5. Manrique-Corredor EJ, Orozco-Beltran D, Lopez-Pineda A, Quesada JA, Gil-Guillen VF, Carratala-Munuera C. Maternal periodontitis and preterm birth: Systematic review and meta- analysis. Community Dent Oral Epidemiol 2019;47:243-51.

6. Mahapatra A, Nayak R, Satpathy A, Pati BK, Mohanty R, Mohanty G, et al. Maternal periodontal status, oral inflammatory load, and systemic inflammation are associated with low infant birth weight. J Periodontol. 2020. 2021;92:1107-16.

7. Figuero E, Han YW, Furuichi Y. Periodontal diseases and adverse pregnancy outcomes: Mechanisms. Periodontol 2000. 2020;83:17588.

8. Rocha JS, Arima L, Chibinski AC, Werneck RI, Moysés SJ, Baldani MH. Barriers and facilitators to dental care during pregnancy: a systematic review and meta-synthesis of qualitative studies. Cad Saude Publica. 2018;34:e00130817.

9. Brasil. Ministério da Saúde (MS). Secretaria de Atenção à Saúde. Departamento de Atenção Básica. Coordenação Nacional de Saúde Bucal. Diretrizes da Política Nacional de Saúde Bucal. Brasília: MS; 2004.

10. Liu PP, Wen W, Yu KF, Gao X, Wong MCM. Dental Care-Seeking and Information Acquisition During Pregnancy: A Qualitative Study. Int J Environ Res Public Health. 2019;16:2621.

11. Sinha S, Bhat PR, Govekar VV, Trasad VA, Acharya AB. Awareness and knowledge regarding maternal periodontal status and associated pregnancy outcomes among the gynecologists of Hubli-Dharwad. J Indian Soc Periodontol. 2020;24:375-8.

12. Vasconcelos $R$, Vasconcelos $M$, Mafra $R$, Queiroz L, Barboza C. Atendimento odontológico a pacientes gestantes: como proceder com segurança. Rev. bras. odontol. 2012;69:120-4.

13. Rocha JS, Arima LY, Werneck RI, Moysés SJ, Baldani $\mathrm{MH}$. Determinants of Dental Care Attendance during Pregnancy: A Systematic Review. Caries Res. 2018;52:139-52.

14. Jain V, Chari R, Maslovitz S, Farine D, Maternal Fetal Medicine Committee, Bujold E, et al. Guidelines for the Management of a Pregnant Trauma Patient. J Obstet Gynaecol Can. 2015;37:553-74.

15. Steinberg BJ, Hilton IV, lida $H$, Samelson R. Oral health and dental care during pregnancy. Dent Clin North Am. 2013;57:195-210. Erratum in: Dent Clin North Am. 2013;57:195-210.

16. Kelaranta A, Ekholm M, Toroi P, Kortesniemi M. Radiation exposure to foetus and breasts from dental X-ray examinations: effect of lead shields. Dentomaxillofac Radiol. 2016;45:20150095.

17. Kanzow P, Wegehaupt FJ, Attin T, Wiegand A. Etiology and pathogenesis of dental erosion. Quintessence Int. 2016;47:275-8. 
18. Takahashi R, Ota E, Hoshi K, Naito T, Toyoshima $\mathrm{Y}$, Yuasa $\mathrm{H}$, et al. Fluoride supplementation (with tablets, drops, lozenges or chewing gum) in pregnant women for preventing dental caries in the primary teeth of their children. Cochrane Database Syst Rev. 2017;10:CD011850.

19. Gil L, Mínguez I, Caffesse R, Llambés F. Periodontal Disease in Pregnancy: The Influence of General Factors and Inflammatory Mediators. Oral Health Prev Dent. 2019;17:69-73.

20. Sharma S, Chandra S, Gupta S, Srivastava S. Heterogeneous conceptualization of etiopathogenesis: Oral pyogenic granuloma. Natl J Maxillofac Surg. 2019;10:3-7.

21.Bi WG, Emami E, Luo ZC, Santamaria C, Wei $S Q$. Effect of periodontal treatment in pregnancy on perinatal outcomes: a systematic review and meta-analysis. J Matern Fetal Neonatal Med. 2021;34:3259-68.

22. Tosounidou S, Gordon C. Medications in pregnancy and breastfeeding. Best Pract Res Clin Obstet Gynaecol. 2020;64:68-76.

23. Poletto VC, Stona $P$, Weber JBB, Fritscher AMGl. Atendimento odontológico em gestantes: uma revisão de literatura. Stomatos. 2008;14:64-75.

24. Rodrigues F, Mármora B, Carrion SJ, Rego AEC, Pospich FS. Anestesia local em gestantes na odontologia contemporânea. J Health NPEPS. 2017;2:254-71.

25. Ouanounou A, Haas DA. Drug therapy during pregnancy: implications for dental practice. $\mathrm{Br}$ Dent J. 2016;220:413-7.

\section{CONFLITO DE INTERESSES}

Os autores declaram não haver conflitos de interesse AUTOR PARA CORRESPONDÊNCIA

Prof. Me. Gerson Aparecido Foratori-Junior Departamento de Odontopediatria, Ortodontia e Saúde Coletiva,

Faculdade de Odontologia de Bauru, Universidade de São Paulo, USP

Bauru - SP, Brasil

Tel: (11) 99766-7202

Email: gerson.foratori@usp.br

Submetido em 22/04/2021

Aceito em 16/07/2021 